\title{
REVIEW
}

\section{Obstetric medicine: Interlinking obstetrics and internal medicine}

\author{
L Acquah, ${ }^{1}$ MD, MSc, FACP; R Burton, ${ }^{2}$ BSc (UK), MB BS (London), PhD (London), MRCOG (UK), FCP (SA), Cert ID Phys (SA) \\ ${ }^{1}$ Mayo Clinic Hospitals, Division of Hospital Internal Medicine, Rochester, Minn, USA \\ ${ }^{2}$ Department of Medicine, Kayelitsha Hospital, Cape Town, and Obstetric Infectious Diseases Clinic, Groote Schuur Hospital, Cape Town, \\ South Africa
}

Corresponding author: $R$ Burton (rosie@polka.co.za)

\begin{abstract}
Medical problems account for almost $50 \%$ of all maternal deaths in South Africa. The most recent report of the National Committee on Confidential Enquiries into Maternal Deaths (NCCEMD) (2008 - 2010) stated that 40.5\% of deaths were due to non-pregnancy-related infections, which are mostly HIV-related, and $8.8 \%$ were due to medical or surgical disorders.

Obstetric physicians have a specific role in managing pregnant and postpartum women with medical problems and, in partnership with obstetricians, can contribute to reducing maternal morbidity and mortality. There are physiological changes in almost all systems in pregnancy. For example, changes in the cardiovascular, respiratory and haematological systems are particularly important when assessing the cause and management of medical problems in pregnant women. Such problems may be unique to pregnancy, exacerbated by pregnancy, or unrelated to pregnancy. They may be present prior to pregnancy, or present for the first time in pregnancy. Some medical problems are worsened by pregnancy. Pregnant women may improve or remain stable, or their disease may predictably or unpredictably deteriorate.

This article discusses the role of obstetric physicians in managing medical problems in pregnant women. A case is described of a pregnant woman with common medical problems, resulting in a serious complication when treatment is interrupted.
\end{abstract}

S Afr Med J 2014;104(9):636-639. DOI:10.7196/SAMJ.8724

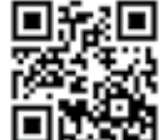

Medical problems account for almost $50 \%$ of all maternal deaths in South Africa (SA). The most recent report of the National Committee on Confidential Enquiries into Maternal Deaths (NCCEMD) (2008 - 2010) stated that $40.5 \%$ of deaths were due to non-pregnancy-related infections, which are mostly HIV-related, and $8.8 \%$ were due to medical or surgical disorders (Table 1). ${ }^{[1]}$ The institutional maternal mortality ratio for SA during this time was 176/100 000 live births, the highest since reporting began in 1998. Maternal deaths have increased with each NCCEMD report, largely because of increasing deaths from HIV/ AIDS. Developed countries such as the USA have a significantly lower maternal mortality ratio. However, this has also increased substantially in recent years, from 6.6/100 000 live births in 1987 to $13.3 / 100000$ live births in 2006. ${ }^{[2]}$

Globally, medical problems in pregnancy are presenting an increasing challenge. In SA, HIV remains the major cause of maternal mortality, and tuberculosis (TB) and other respiratory infections are the most common causes of death. However, non-HIV-related medical problems are increasingly significant. In many developing countries, postpartum haemorrhage secondary to an atonic uterus is the most common cause of death. ${ }^{[3]}$ Pulmonary embolism causes most maternal deaths in resource-rich countries. ${ }^{[4]}$

Confidential Enquiries into Maternal Deaths allow avoidable factors to be analysed and corrective measures to be implemented. Common avoidable factors include lack of problem recognition, substandard care, disparity in healthcare resources, inequity in access to care and lack of expertise among healthcare workers. ${ }^{[5]}$ Many doctors working in maternity units have little training or experience in internal medicine. Obstetrics and Gynaecology is primarily a surgical specialty, with neither undergraduate nor postgraduate training including medical problems in pregnancy. Equally, internal medicine programmes do not address pregnancy; therefore doctors working in this field - from interns to consultants - have little exposure to pregnant women. Obstetric physicians are internal medicine specialists who have training and experience in managing 
Table 1. Causes of maternal mortality in South Africa (adapted from Saving Mothers 2008 - 2010 ${ }^{[1]}$ )

\begin{tabular}{ll}
\hline Causes & $\%$ \\
\hline Direct maternal deaths & $46.3^{*}$ \\
Obstetric haemorrhage & 14.0 \\
Hypertension & 14.0 \\
Pregnancy-related sepsis & 5.3 \\
Embolism & 1.9 \\
Indirect maternal deaths & $49.3^{*}$ \\
Non-pregnancy-related infections & 40.5 \\
HIV-positive & 87.3 \\
HIV-negative & 5.2 \\
Unknown, or declined testing & 7.5 \\
Major subcategories & \\
$\quad$ Tuberculosis & 26.9 \\
Other pneumonia & 26.7 \\
Pneumocystis jirovecii pneumonia & 13.3 \\
Cryptococcal meningitis & 4.2 \\
Other meningitis & 8.7 \\
Medical and surgical disorders & \\
Major subcategories & \\
Africa. Non-pregnancy-related infections, which are mostly HIV-related, greatly \\
sutnumber the four most common direct causes of death. Note: Only selected causes are \\
×Of all maternal deaths.
\end{tabular}

pregnant women with medical problems. In partnership with obstetricians, they have a significant role in optimising management and can contribute to reducing maternal morbidity and mortality from medical causes. ${ }^{[6,7]}$

\section{What is obstetric medicine?}

Obstetric medicine is a specialty of internal medicine that focuses on managing the medical problems of women with significant comorbidities that may exist prior to pregnancy, or arise during pregnancy or in the puerperium. There are physiological changes in almost all systems in pregnancy. For example, changes in the cardiovascular, respiratory and haematological systems are

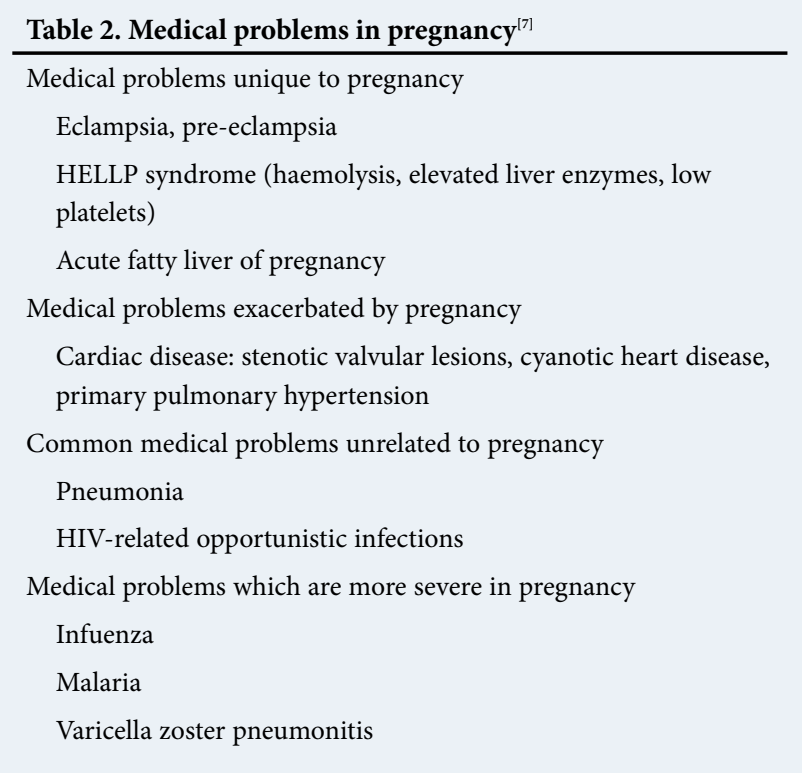

particularly important when assessing the cause and management of medical problems in pregnant women. ${ }^{[7]}$

Obstetric physicians work with obstetricians and other physicians to co-ordinate and comprehensively manage the medical problems of vulnerable pregnant and postpartum women. Obstetric medicine is an established sub-specialty of internal medicine in Australia. Other countries, including the USA and Canada, where there are formal training programmes in this field, are working towards accreditation. Since there are few obstetric physicians in most countries, those available play an important role in training other physicians, obstetricians, non-specialist doctors and midwives.

Medical problems in pregnancy may be unique to pregnancy, exacerbated by pregnancy or unrelated to pregnancy. They may be present prior to pregnancy or present for the first time during pregnancy. Some medical problems are worsened by pregnancy (Table 2). ${ }^{[7]}$ Pregnant women may improve or remain stable, or their disease may predictably or unpredictably deteriorate. The expected changes in maternal physiology, baseline disease characteristics and extent to which treatment is optimised are all important factors in determining how a specific disease will respond to pregnancy. Medical problems may also affect pregnancy outcomes, with increased risk of preterm delivery, growth restriction, stillbirth and neonatal loss. The best time to ensure optimal management of chronic medical problems is before pregnancy. However, women are often given no advice on whether pregnancy may worsen their medical condition, or whether their medical problem is likely to cause complications during pregnancy. Medical advances have significantly enhanced the life 
expectancy and quality of life of many patients with chronic diseases, including HIV infection. Women whose medical problems would previously have resulted in premature death or severe disability now want to live 'normal lives', and desire children. As maternal age and the prevalence of obesity increase, it is likely that common comorbidities such as cardiovascular disease, chronic hypertension and diabetes mellitus may become increasingly prevalent in pregnant women, and contribute towards increasing maternal morbidity and mortality.

The case study below involves common medical problems. However, their management in pregnancy can be challenging, and without optimal treatment may lead to serious complications.

\section{Case study}

A 28 -year-old woman presented to her clinic at 20 weeks' gestation with her first pregnancy. She had the following baseline medical problems:

- diabetes mellitus type 1 , poorly controlled diabetes, and a random finger prick glucose of $16 \mathrm{mmol} / \mathrm{L}$

- chronic kidney disease secondary to diabetes, pre-pregnancy glomerular filtration rate $40 \mathrm{~mL} / \mathrm{min}$, with $3 \mathrm{~g}$ proteinuria/24 h

- chronic hypertension - also poorly controlled, and a blood pressure (BP) of 150/100 $\mathrm{mmHg}$

- HIV-negative on testing on day of presentation.

She has not received any pre-pregnancy counselling. She would have greatly benefited from good control of her diabetes and hypertension prior to pregnancy and a thorough discussion of the risks both she and her infant may encounter during her pregnancy.

Gradually, she has become heat intolerant with associated palpitations and irritability. Her examination revealed a highly anxious diaphoretic woman, with tachycardia at 140 beats/minute, BP 155/96 mmHg, asymmetrical proptosis, fine tremors and an enlarged, palpable thyroid. She was transferred to the obstetric ward of her local hospital and reviewed by the obstetric team and an obstetric physician.

She needed optimal control of her diabetes and hypertension, and close monitoring of both for the duration of her pregnancy. The patient was at increased risk of pre-eclampsia owing to her diabetes and hypertension, and of deteriorating renal function because of her diabetic nephropathy. Fetal risks of diabetes in pregnancy include congenital abnormalities, macrosomia, intrauterine death, respiratory distress syndrome and neonatal hypoglycaemia.

She developed symptoms and signs of thyrotoxicosis, confirmed with thyroid function tests that showed thyroid-stimulating hormone (TSH) $0.01 \mathrm{mU} / \mathrm{L}$ and T4 of $43 \mathrm{pmol} / \mathrm{L}$. Many clinical features are common in normal pregnancy, including heat intolerance, tachycardia, palpitations and a palpable thyroid. However, symptoms and signs that support hyperthyroidism include tremor, persistent tachycardia, weight loss and lid lag. Exophthalmos persists after treatment and therefore does not confirm new hyperthyroidism in the absence of other features.

She was diagnosed with Graves' hyperthyroidism, the most common cause of hyperthyroidism during pregnancy. ${ }^{[7-9]}$ TSH receptor-stimulating antibodies (TRAbs) were positive. There are less common causes of thyrotoxicosis, including multinodular goitre and subacute thyroiditis. There are also uncommon causes that are specific to pregnancy, because the alpha sub-unit of the beta human chorionic gonadotropin (hCG) molecule can mimic TSH. These include hyperemesis gravidarum that has high beta hCG levels that peak at $10-12$ weeks' gestation, and which was therefore unlikely in this patient. Trophoblastic hyperthyroidism may also occur. An ultrasound scan did not reveal a complete hydatidiform mole or other findings consistent with gestational trophoblastic disease.

She was started on high-dose carbimazole and propranolol. Carbimazole crosses the placenta, and can cause fetal hypothyroidism and goitre, and rarely a fetal abnormality called aplasia cutis, in which there are patches of absent skin on the scalp. Short-term use of propranolol is safe in pregnancy. She was discharged to follow up at an obstetric medicine clinic.

At 24 weeks' gestation she developed severe hypertension, worsening proteinuria and deteriorating renal function. She was admitted to the antenatal ward, where the admitting doctor discovered that she was taking her carbimazole and propranolol erratically. She also missed her appointments due to worsening fatigue, and the clinic staff did not have a policy in place to follow up women who have defaulted. She had not been taking her treatment for hypertension, and had not been monitoring her blood sugar and increasing her doses of insulin. One week after admission to restart and maintain her on adequate doses of her medications, she improved and was discharged home. Unfortunately, she did not attend further clinic appointments.

At 28 weeks' gestation she presented as an emergency case to her obstetric unit. She had a productive cough with haemoptysis and dyspnoea, severe nausea with vomiting, and agitation with psychotic features. She had pain in her left leg and thigh. Her vital signs were as follows: temperature $39-40^{\circ} \mathrm{C}$, pulse 140 beats/minute at an irregular rate and rhythm, oxygen saturation on room air $85 \%$, respiratory rate 30 breaths/minute, and BP 160/110 mmHg. She appeared jaundiced and could not sit still. She was highly impatient with caregivers and had pressured speech. She had fair air entry with diffuse wheezes throughout her lungs. Extremities revealed 3 - 4+ pitting oedema from feet to thighs. She had peri-orbital oedema, jaundice, and proptosis with lid lag. Her jugular veins were distended, she had a third heart sound and a flow murmur. Her right upper abdominal quadrant was tender on mild palpation. She had no nystagmus, but had hyperreflexia with 4 beats of clonus. Her skin was warm and wet without a rash. At this point creatinine was $220 \mu \mathrm{mol} / \mathrm{L}$, with $7 \mathrm{~g}$ protein $/ 24 \mathrm{~h}$. 


\section{What is the most likely diagnosis?}

- Severe pre-eclampsia with lower respiratory tract infection or TB?

- Venous thromboembolism: acute deep venous thrombosis of the left lower extremity and pulmonary embolism?

- Worsening diabetic nephropathy, or an additional cause of deteriorating kidney function?

- Thyroid crisis (thyroid storm)?

- Congestive heart failure secondary to a thyroid crisis?

She had developed a severe life-threatening condition, i.e. a thyroid storm (an exaggerated thyrotoxicosis), which was precipitated by her uncontrolled Graves' hyperthyroidism. ${ }^{[10,11]}$ She had acute cardiorespiratory distress (congestive cardiac failure with atrial fibrillation, pulmonary oedema, haemoptysis and peripheral oedema). She also had thermoregulatory changes (hyper-pyrexia), gastrointestinal involvement (jaundice, nausea, vomiting), and central nervous system dysfunction (severe agitation, psychosis). There was no evidence of deep vein thrombosis. She needed urgent management by her multidisciplinary team, which includes an obstetrician, junior obstetric doctors, an obstetric physician, an endocrinologist and midwives. If she was at a level 1 hospital without physicians on site, telephonic consultation was indicated. She needed referral when stabilised (to a high-care or an intensive care unit).

Thyroid crisis and congestive cardiac failure in pregnancy are managed in the same manner as in non-pregnant patients. Her cardiac failure needed immediate medical management and possibly intubation. In terms of her thyroid crisis, she needed beta-blockade, e.g. propranolol 60 - $80 \mathrm{mg}$ orally every 4 - 6 hours. Controlling her tachycardia would improve her cardiovascular function. Beta-blockade could worsen her cardiac failure owing to negative ionotropic effects, and therefore had to be administered extremely cautiously. She was given carbimazole, with Lugol's iodine (potassium iodine-iodide solution) started at least one hour later. Administration of iodine was delayed for one hour so that the iodine would not be utilised by the thyroid gland to synthesise new thyroid hormone.

She might have a polyglandular deficiency due to her diagnosis of Graves' hyperthyroidism and diabetes mellitus, i.e. potential hypoadrenalism. Therefore, her severe life-threatening autoimmune condition might benefit from glucocorticoids, e.g. hydrocortisone $100 \mathrm{mg}$ every 8 hours. ${ }^{[12]}$
She responded well to treatment, and was discharged home a week later. She is now 29 weeks pregnant, and still faces significant maternal and fetal risks from her diabetes, nephropathy, hypertension and hyperthyroidism. She needs close follow-up for the remainder of her pregnancy, involving both obstetricians and physicians. A multidisciplinary obstetric medicine clinic would be ideal; at each consultation, her medical problems, obstetric problems and potential fetal complications can be reviewed. Timing and mode of delivery will need to be discussed and paediatricians will also need to be involved. Even if she delivers at term without further complications, her baby will need neonatal unit admission for hypoglycaemia monitoring. There is also a risk of fetal or neonatal thyrotoxicosis due to transplacental passage of TRAbs.

\section{References}

1. National Committee on Confidential Enquiries into Maternal Deaths. Saving Mothers 2008 - 2010: Fifth Report on the Confidential Enquiries into Maternal Deaths in South Africa. Pretoria: National Department of Health, 2012. http://www.health.gov.za/docs/reports/2012/savingmothersexec.pdf (accessed 21 July 2014).

2. World Health Organization. Trends in Maternal Mortality: 1990 to 2008 Estimates Developed by WHO, UNICEF, UNFPA and the World Bank. Geneva: World Health Organization, 2010. http:// whqlibdoc.who.int/publications/2010/9789241500265_eng.pdf (accessed 19 July 2014).

3. World Health Organization. Mother-Baby Package (WHO/RHT/MSM/94.11, Rev1). Geneva: World Health Organization, 1998.

4. Bourjeily G, Paidas M, Khalil H, Rosene-Montella K, Rodger M. Pulmonary embolism in pregnancy. Lancet 2010;375(9713):500-512. [http:dx.doi.org/10.1016/S0140-6736(09)60996-X]

5. Silal SP, Harris B, Birch S, McIntyre D. Exploring inequalities in access to and use of maternal health services in South Africa. BMC Health Serv Res 2012;12:120. [http://dx.doi.org/10.1186/1472-696312-120]

6. Nelson-Piercy C, Mackillop L, Williams DJ, Williamson C, de Swiet M, Redman C. Maternal mortality in the UK and the need for obstetric physicians. Br Med J 2011;343:d4993. [http://dx.doi.org/10.1136/ bmj.d4993]

7. Nelson-Piercy C. Handbook of Obstetric Medicine. 4th ed. London: Informa Health Care, 2010.

8. Neale D, Burrow G. Thyroid disease in pregnancy. Obstet Gynecol Clin North Am 2004;31(4):893-905. [http://dx.doi.org/10.1016/j.ogc.2004.09.001]

[http://dx.doi.org/10.1016/j.ogc.2004.09.001]
Stagnaro-Green A (Chair), Abalovich M, Alexander E, et al. and the American Thyroid Association Stagnaro-Green A (Chair), Abalovich M, Alexander E, et al. and the American Thyroid Association
Taskforce on Thyroid Disease During Pregnancy and Postpartum. Guidelines of the American Thyroid Association for the Diagnosis and Management of Thyroid Disease During Pregnancy and Postpartum. Thyroid 2011;21(10):1081-1225. [http://dx.doi.org/10.1089/thy.2011.0087]

10. Burch HB, Wartofsky L. Life-threatening thyrotoxicosis. Thyroid storm. Endocrinol Metab Clin North Am 1993;22:263.

1. Nayak B, Burman K. Thyrotoxicosis and thyroid storm. Endocrinol Metab Clin North Am 2006;35:663686. [http://dx.doi.org/10.1016/j.ecl.2006.09.008]

2. Tsatsoulis A, Johnson EO, Kalogera $\mathrm{CH}$, Seferiadis $\mathrm{K}$, Tsolas $\mathrm{O}$. The effect of thyrotoxicosis on adrenocortical reserve. Eur J Endocrinol 2000;142(3):231. [http://dx.doi.org/10.1530/eje.0.1420231]

\section{Resources in Obstetric Medicine}

- Reference 7 is a practical guide to investigation and management of medical problems in pregnancy.

- Journal: Obstetric Medicine: The Medicine of Pregnancy: obmed.rsmjournals.com

- International Society of Obstetric Medicine: http://www.isomnet.org 